Published in final edited form as:

Transpl Int. 2009 January ; 22(1): 38-48. doi:10.1111/j.1432-2277.2008.00728.x.

\title{
MINIMIZATION OF STEROIDS IN KIDNEY TRANSPLANTATION
}

\author{
Arthur J. Matas, MD \\ Department of Surgery, University of Minnesota, 420 Delaware St. SE, Minneapolis, MN 55455, \\ (612) 625-6460
}

Arthur J. Matas: matas001@umn.edu

\section{Abstract}

The goal of steroid minimization trials has been to minimize or eliminate steroid-related side effects while simultaneously not increasing the rate of acute rejection (AR) and chronic graft loss. Early trials of late steroid withdrawal ( $\geq 3$ months posttransplant) were associated with significantly increased AR rates and late graft loss. More recent trials of rapid discontinuation of prednisone (RDP) ( $\leq 7$ days posttransplant) have been associated with little or no increase in AR rates and no difference in graft survival (versus maintenance prednisone). Of note, induction therapy appears to be important for success; however, it is not clear if any single maintenance protocol is superior.

Intermediate-term follow-up (5-7 years) is now available for some randomized and nonrandomized trials; graft survival and renal function remain excellent. Most of these trials have been done in low immunologic risk recipients, but there are reports of success of RDP in children, blacks, sensitized recipients, recipients with potentially recurring disease, and kidney-pancreas recipients. Of critical importance, steroid-related side effects have been minimized.

Steroid minimization protocols can clearly be recommended for the low risk patient; additional trials are necessary for those at higher risk. Additional research is also necessary on integrating calcineurin minimization with steroid minimization.

The goal of steroid minimization protocols has been to eliminate or minimize steroid-related side effects while not increasing the rates of acute rejection (AR) or chronic graft loss. Until recently, corticosteroids had been a mainstay of kidney transplant immunosuppressive protocols. However, though inexpensive, steroids are associated with debilitating side effects, including hypertension, hyperlipidemia, cataracts, avascular necrosis, osteoporosis, mood and appearance changes, and, in children, growth retardation (1). Vanrenterghem et al. recently showed that increased long-term total steroid dose is associated with increased cardiovascular morbidity (2). Treatment of these steroid-related side effects adds to the cost of transplants (3). In addition, such side effects increase posttransplant noncompliance (4); noncompliance is associated with an increased incidence of AR, chronic rejection, and graft loss (5). Thus, a hidden cost of steroid-related side effects may be increased graft loss. When surveyed, kidney transplant recipients state that the immunosuppressive drug they would most like not to take is prednisone (6).

\section{Late ( $\geq 3$ month) steroid withdrawal}

Historically, numerous attempts have been made either to avoid steroids or, in selected recipients, to gradually withdraw steroids late ( 23 months) posttransplant. After cyclosporine (CSA) was introduced, CSA monotherapy was associated with a high incidence of AR $(7,8)$. Similarly, meta-analyses of studies of late steroid withdrawal in selected recipients on CSA and prednisone (with or without azathioprine [AZA]) showed an increased incidence of AR and graft loss $(9,10)$. 
Of particular concern has been a multicenter Canadian study in which recipients on CSA and prednisone were randomized at 3 months to either switch to CSA monotherapy or continuing the 2 drugs (11). For the first 500-600 days after randomization, that study's authors found to significant differences between the 2 groups; but thereafter, the CSA monotherapy group had an increased rate of graft loss. The Canadian study has led to ongoing concerns that even if steroid minimization protocols have early success, late graft failure will significantly increase. Yet it is critical to realize that this study was done before the impact of AR on long-term graft outcome was recognized (12); the authors did not determine whether or the rate of AR increased after prednisone withdrawal.

After studies showing mycophenolate mofitil (MMF) was associated with a lower AR rate than AZA, randomized studies of late steroid withdrawal were done in selected recipients on CSA and MMF, and subsequently in selected recipients on tacrolimus (TAC) and MMF (13-18). Recent meta-analyses of those studies (4 used CSA, 2 TAC) showed a significantly increased AR rate in the steroid withdrawal group, but no increased risk of early graft failure $(19,20)$. Longer-term follow-up has only been reported for 1 of those 6 studies $(21)$, a TACMMF study in which 3- year follow-up showed no difference between the steroid withdrawal and the maintenance immunosuppression groups in patient and graft survival rates or in renal function; the steroid withdrawal group had less hypertension and significantly lower mean total cholesterol and LDL-cholesterol values $(\mathrm{p}=.02)$.

In contrast to the above studies, Opelz et al recently reported no increased AR rates for recipients on CSA-based immunosuppression who underwent steroid withdrawal $>6$ months posttransplant (22). Median time to steroid withdrawal was 1.1 years; after enrollment, steroids were tapered in step-wise fashion. Using the Collaborative Transplant Study (CTS) database, Opelz et al matched each enrolled recipient $(n=1015)$ with 3 controls. The actuarial 7-year patient, graft, and death-censored graft survival rates were significantly better for the withdrawal group ( $\mathrm{p}<.01)$. Outcomes did not differ for those treated with AZA or MMF. The difference in outcomes between Opelz's vs. the above studies may be due to the later withdrawal of steroids in Opelz's study.

Two randomized trials using calcineurin inhibitors recently published their results (not included in the above meta-analyses). Pelletier et al randomized selected CSA-MMFprednisone recipients $>6$ months posttransplant, to either withdraw or continue prednisone (23); the long-term outcome of the 2 groups did not significantly differ. Wlodarczyk et al. randomized recipients, at the time of transplant, to take either TAC-MMF-prednisone or TAC-AZA-prednisone (24). At 3 months posttransplant, selected recipients (i.e, those that were then rejection-free and having had no more than 1 steroid-sensitive rejection in the first 2 months; serum creatinine level $<160 \mu \mathrm{mol} / \mathrm{L}$ between days 84 and 91 posttransplant; receiving at least $0.5 \mathrm{gm} / \mathrm{day}$ of $\mathrm{MMF}$ or $1 \mathrm{mg} / \mathrm{kg} /$ day of AZA) were randomized to either taper off or stay on steroids. For both the TAC-MMF and TAC-AZA groups, the rejection rate in the next 3 months was similar to the rate in each group randomized to stay on steroids.

Success with steroid withdrawal $>3$ months posttransplant has also been reported with mTOR inhibitors. Buchler et al. reported a multicenter study in which recipients were randomized, at the time of their transplant, to take either sirolimus (SRL) or CSA (25). All were treated with antithymocyte globulin (ATG), MMF, and a 6-month course of corticosteroids. Within 48 hours posttransplant, SRL recipients received a 15 -mg loading dose for 2 days, followed by $10 \mathrm{mg} /$ day (target, 10 to $15 \mathrm{ng} / \mathrm{mL}$ ). At 12 months posttransplant ( 6 months after steroid withdrawal), the patient and graft survival rates, the incidence of biopsy- proven rejection, and the rate of steroid withdrawal did not significantly differ between the 2 groups. At 12 months, for recipients who remained on 
their protocol, the estimated glomerular filtration rate (GFR) was significantly higher with SRL $(69 \pm 19 \mathrm{ml} / \mathrm{min})$ than with CSA $(60 \pm 14 \mathrm{ml} / \mathrm{min})(P=0.01)$. However, study drug discontinuation rates were higher with SRL (28.2\%) than with CSA (14.9\%). Adverse events were also much higher with SRL. Buchler et al. felt that avoiding a loading dose of SRL and delaying the introduction of SRL during antibody induction might have prevented many of the SRL-related complications. Both Mahalati and Kahan (26) and Citterio et al (27) have reported successful late steroid withdrawal for recipients on CSA plus SRL.

In an interesting nonrandomized study, Hricik and colleagues reported their results of late prednisone withdrawal in 30 African American kidney transplant recipients on TAC-SRL (28). With a mean follow up of 14 months, the AR rate was only $6.7 \%$ (28); but during longer follow-up (mean 48.5 months), the cumulative incidence of rejection was $41 \%$, and graft loss occurred in $25 \%$ of recipients. Of importance, 9 of the 13 rejection episodes were related to noncompliance (29).

Of note, most (but not all) studies reporting success of late steroid withdrawal have been done in Europe; most studies (but not all) reporting failure have been done in the United States. It may be that differences in organ allocation systems, population demographics, or timing of withdrawal explain these outcome differences.

\section{Rapid Discontinuation of Prednisone (RDP)}

The recognition that late steroid withdrawal was associated with increased AR rates, combined with the introduction of newer and more potent induction and maintenance immuno-suppressive agents led many investigators to consider either rapid discontinuation of prednisone ( $\leq 7$ days posttransplant) (RDP) or complete avoidance of steroids. Such protocols have the potential advantage of minimizing any early steroid-related side effects. Both RDP and avoidance protocols have used a variety of induction agents (Thymoglobulin, Alemtuzumab, interleukin-2 inhibitors), either of the 2 calcineurin inhibitors (CSA or TAC), and either MMF or SRL.

Birkeland was the first to report success with steroid avoidance (Table 1) $(30,31)$. In his series of 100 recipients (67 deceased donor, 33 living donor), recipients were on with ATG, cyclosporine, and MMF. Only $13 \%$ had an AR episode; the actuarial 4 year graft survival rate was $82 \%$.

\section{1) Randomized studies of RDP}

a) Short-term ( $\leq 1$ year) results-Since Birkeland's report, a number of prospective, randomized trials of RDP vs. maintenance prednisone have been done (Table 2) (32-43).

Almost all have used antibody induction. Most have limited the protocol to recipients with a relatively low immunologic risk; an exception was the study by ter Meulen et al. $(n=364)$ in which the only exclusion criteria were use of an HLA-identical living donor and treatment with prednisone at the time of the transplant (33). In general, short-term results of these studies have shown no difference between RDP and maintenance prednisone groups in patient or graft survival or AR rates, severity of AR or in renal function (Table 2).

In contrast to others, Vincenti et al recently reported the 12-month outcome of a trial in which recipients $(\mathrm{n}=357)$ on basiliximab-CSA and enteric-coated mycophenolate sodium were randomized to 1 of 3 groups: no prednisone, vs. prednisone till day 7 posttransplant, vs. maintenance prednisone (37). Biopsy-proven AR rates were significantly increased in the 2 prednisone minimization groups vs. the maintenance group. When recipients with graft loss or death were assigned a GFR of $0 \mathrm{ml} / \mathrm{min}$, the mean 12-month GFR was lower in the 2 
prednisone minimization groups vs. the prednisone maintenance group. Per a subset analysis of recipients with functioning grafts at 12 months, GFR did not differ between groups.

In the above studies, RDP was done in the context of induction plus 2 maintenance drugs. In a different trial design, Vitco et al randomized 471 recipients to triple therapy (TAC-MMFprednisone), vs. TAC-MMF, vs. basiliximab-TAC (35). The 2 prednisone-free groups had significantly increased AR rates (TAC-MMF, 30.5\%; basiliximab-TAC, 26.1\%) vs. the triple therapy group $(8.2 \%)$ ( $<<.001)$. But the groups did not significantly differ in patient or graft survival rates. Vitco's study suggests that both induction and dual-agent maintenance therapy may be important for successful RDP.

b) Intermediate-term ( $>1$ year) follow-up-Woodle et al. randomized 386 recipients (stratified by donor source and recipient race) on antibody induction (center choice) and TAC-MMF to either RDP or long-term prednisone (39). At 3 years, the primary composite endpoint of death, graft loss, or severe AR did not differ between groups. Nor did renal function (mean creatinine; calculated creatinine clearance). The RDP group had a significant lower rate of diabetes and fractures; their triglycerides values were lower and their hypertension easier to control. Although not statistically significant, there was a $6.5 \%$ increased AR rate in the RDP group ( $\mathrm{p}=.07$ ); in addition, the rate of chronic allograft nephropathy (biopsy for cause) was 5\% higher in that group ( $\mathrm{p}=\mathrm{NS}$ ). Of note, in a subgroup analysis, RDP recipients on Thymoglobulin had a lower AR rate than those on IL-2r inhibitors.

Montagnino et al. randomized 133 de novo kidney transplant recipients on basiliximab, everolimus, and CSA to either RDP or long-term low-dose steroids (40). During the followup, $46 \%$ in the RDP group subsequently resumed prednisone. Per to the intention-to-treat analysis, the 3-year graft survival rate was $95 \%$ in the RDP arm vs $87 \%$ in the steroid arm $(P=\mathrm{NS})$. The RDP group had more biopsy-proven rejections (32\%) than the steroid group (18\%), but the difference was only of borderline significance $(\mathrm{p}=0.059)$. After 3 years, the 2 groups did not differ in their mean creatinine clearance, mean serum cholesterol, and mean triglyceride levels.

Kumar et al. randomized 300 recipients treated with basiliximab, a calcineurin inhibitor, and either MMF or SRL, to either RDP or maintenance prednisone (41). Their study began as a randomized study; however, an after interim analysis showed the benefit of RDP, it was then continued, with IRB approval, as a nonrandomized study (patients were informed of the results of the interim analysis). The 2 groups did not differ in 3-year patient and graft survival rates, in AR rates, in mean serum creatinine level and creatinine clearance, the incidence of subclinical rejection, or in the progression of chronic allograft nephropathy. At 3 years, the RDP group had a significantly lower rate of new onset diabetes mellitus ( $\mathrm{p}<.01$ ), less increase in BMI $(\mathrm{p}<.04)$, and fewer infections requiring hospitalization $(\mathrm{p}=.05)$. Outcome did not significantly differ between those receiving SRL vs. MMF (see below) (42).

In a pilot study with a differing trial design, Boots et al randomized TAC-MMF recipients to either RDP or steroid tapering and withdrawal at 3 to 6 months (median 2.7 years follow-up) (43). No induction therapy was used. The groups did not differ significantly in patient or graft survival rates or in renal function. Of note, AR episodes occurred in $29 \%$ of the RDP group and $30 \%$ of the withdrawal group. 


\section{2) Nonrandomized studies}

a) short-term ( $\leq 1$ year) results-Cole et al reported a pilot study of 57 recipients on daclizumab, CSA, and MMF who underwent RDP (44). At 1 year, the patient survival rate was $95 \%$; graft survival, $89 \%$. Of the 57 recipients, 14 (25\%) had an AR episode.

Rajab et al. compared outcome for 301 first transplant recipients on ATG induction, CSA, SRL, and RDP vs. for historical controls (502 first transplant recipients in the 2 years before institution of RDP) on basiliximab induction, CSA, MMF, and prednisone (45). The 1-year patient survival and death-censored graft survival rates, the mean serum creatinine level, and the mean serum triglyceride and cholesterol values did not differ significantly between the groups. The biopsy-proven AR was $4.9 \%$ rate in the RDP group and $9.4 \%$ in the control group $(P<0.01)$; weight gain was also significantly lower in the RDP group. However, more recipients in the RDP group required erythropoietin and iron therapy for anemia $(P<0.001)$.

Woodle et al reported the results of a pilot study in 77 recipients on basiliximab, SRL, TAC, and RDP (46). At 12 months posttransplant, the patient and graft survival rates were each $100 \%$. The biopsy-proven AR rate was $13 \%$; the clinically diagnosed AR rate, an additional $10.5 \%$.

Kato et al. compared outcome for recipients on basiliximab and CSA-MMF who underwent RDP with concurrent recipients on CSA-MMF and long-term prednisone (47). At 1 year posttransplant the patient survival, graft survival, and AR rates did not differ between groups. Kato et al noted that for those successfully prednisone-free at 1 year, there was no need to subsequently restart prednisone

b) Intermediate-term ( $>1$ year) follow-up_Jaber et al. reported their experience with 3 agent-immunosuppression involving RDP and calcineurin-inhibitor minimization $(\mathrm{n}=84)$ (48). Immunologically high-risk recipients were included. ATG was given for 5 days, together with TAC- MMF. SRL was initiated on day 6 posttransplant, when prednisone was discontinued. As compared with historical controls, the study group's 2.5 -year actuarial patient survival rate $(97 \%)$ was significantly higher $(\mathrm{p}=0.048)$; there was no difference in graft survival (93\%), acute rejection-free graft survival (89\%), or renal function. In addition, posttransplant, the study group had a decreased prevalence of cardiovascular disease risk factors, as compared with controls.

We began RDP at our institution in 1999 (49), and have reported 6-year outcome ( $\mathrm{n}=775$ ) (50). Our first and second transplant recipients are treated with ATG, a CNI, either MMF or SRL, and RDP. Our only exclusions were recipients already on prednisone. The actuarial 6year patient survival rate was $88 \%$; graft survival, $80 \%$. At 1 year, $13 \%$ of recipients had had an AR episode. Renal function (MDRD GFR was stable through 6 years. Of note, $8 \%$ of recipients developed cytomegalovirus (CMV) infection; $0.5 \%$, polyomavirus. We found that over $80 \%$ of recipients remained prednisone-free long-term.

In another study, we compared outcome for recipients who underwent RDP vs. historical controls on ATG, calcineurin inhibitor, MMF, and long-term prednisone (51). The actuarial patient survival, graft survival, AR, or biopsy-proven interstitial fibrosis and tubular atrophy (IF/TA) rates did not differ significantly between the 2 groups; nor did the MDRD GFR.

The RDP group had significantly lower rates of cataracts ( $<.001)$, new onset posttransplant diabetes $(\mathrm{p}<.001)$, avascular necrosis $(\mathrm{p}<.001)$, cytomegalovirus infection $(\mathrm{p}<.001)$, fractures $(\mathrm{p}=.04)$, and non-PTLD malignancy $(\mathrm{p}=.02)$

Gallon et al. reported the outcome of a sequential study in which recipients on IL-2r induction were either maintained on chronic prednisone $(n=96)$ or underwent RDP $(n=116)$ 
(52). The 2 groups did not differ in actuarial 7 -year patient and graft survival rates, the incidence and severity of acute cellular rejection, and the slope of GFR decline per month 5 years posttransplant. The RDP group had a significantly lower incidence of hyperlipidemia and posttransplant diabetes.

\section{Control groups --- a moving target}

Late steroid withdrawal and RDP protocols were developed in the context of what, today, would be considered relatively high long-term prednisone doses. Thus, the validity of comparing outcome to historical controls has been questioned. Certainly, withdrawal and RDP protocols have helped move the transplant field forward by helping demonstrate that high long-term steroid doses are not necessary. Today, even recipients maintained on longterm prednisone are taking far less prednisone than they would have taken a decade ago. For example, recipients at our institution not on our RDP protocol (e.g., those on prednisone at the time of transplant) are discharged to home on only $5 \mathrm{mg} /$ day of prednisone.

However, given that prednisone has an anti-inflammatory effect, concern remains that the rate of chronic graft loss will significantly increase in recipients on RDP protocols. To date, this concern is supported by no data. In addition, it has been suggested that most steroidrelated side effects are related to long-term high doses and that the risk-benefit ratio may be far different with long-term low doses. However, van den Ham et al showed a significant difference in weight gain between recipients on $5 \mathrm{mg}$ /day of prednisone vs. RDP (53). Matsunami et al. noted that recipients on high-dose steroids had a $55 \%$ incidence of posterior subcapsular cataracts; low dose steroids, 28\%; and no steroids, $6.2 \%$ (54). Steroids have been associated with rapid loss of bone mineral density in transplant recipients (55) and cumulative steroid dose has been correlated with bone mineral density loss (56). In addition, considerable data from the nontransplant literature showed that even a short course of low-dose prednisone is associated with significant loss of bone mineral density and with a significant increased fracture rate (57-61).

\section{Individual populations on RDP}

Most of the above studies limited RDP to adult recipients with a relatively low immunologic risk. However, some randomized and some nonrandomized studies have enrolled both highand low- risk recipients $(33,41,47,51)$. Only a few reports have described outcome of RDP in specific higher-risk populations.

a. Children - Successful steroid avoidance in children was first shown by Birkeland et al. using ATG induction, MMF, and CSA(n=14); the early AR rate was $15 \%$ (62). Subsequently, Sarwal et al. described a novel steroid avoidance protocol, based on TAC-MMF, and daclizumab induction for 6 months posttransplant $(63,64)$. In 57 consecutive (low sensitization risk) recipients, AR rates were low (8\%); there was excellent graft function and dramatic catchup growth trends. The RDP group had better estimated creatinine clearance, less hypertension, a lower increase in BMI, and, importantly, better compliance as compared with historical controls. In a retrospective case-controlled study, Oberholzer et al. noted that their RDP group had significantly higher creatinine clearance at 6 and 12 months posttransplant ( $\mathrm{p}=$. 04), significantly lower BMI, and significantly higher delta Z score, and significantly less hyperlipidemia, body disfigurement, and need for antihypertensive medications (65).

Chavers et al compared 21 children ( $14 \pm 3 \mathrm{yrs}$ ) on an RDP protocol with 39 matched controls on maintenance prednisone and found no difference in patient or 
graft survival, AR rates, anemia, hypertension, creatinine level, or CMV or EBV infection (66). Of the RDP group, $82 \%$ remained prednisone-free.

b. Blacks- Blacks have had an increased rate of AR episodes on late steroid withdrawal protocols (12). Thus, there has been concern that early prednisone minimization would also result in an increased AR rate. However, data to date suggests that early steroid minimization can be successful in blacks (67-70).

Haririan et al. compared blacks treated with RDP vs. blacks on chronic steroid therapy: the 1-year patient and graft survival and AR rates did not differ between groups (67). The RDP group had less weight gain, lower cholesterol levels at 3 months, and a lower risk of posttransplant diabetes mellitus. Others compared their 1 -year results for blacks vs. nonblacks in their RDP trials and found no difference in patient and graft survival rates, AR rates, or in renal function (68-70). However, Kumer et al found that subclinical rejection at 1 month was significantly higher in blacks ( $P=0.04)$ (69). Kumar et al recently reported their 5 -year outcome (71). Of note, in their series blacks (vs. nonblacks) had a higher percentage of deceased donor transplants and of recipients with diabetes, and had a longer duration of pretransplant dialysis. There was no difference between the 2 groups in 5 year graft survival; however, blacks had a significantly increased subclinical rejection at 3and at 5-years, significantly more tubular atrophy and interstitial fibrosis and a significantly lower GFR at 5 years.

c. potentially recurrent disease - Ibrahim et al. studied outcome for 105 adult recipients whose transplant was for glomerulonephritis (GN) and who underwent RDP (72). Two control groups consisted of: a) 439 concurrent recipients whose transplant was for causes other than GN and who underwent RDP, and b) 260 recipients whose transplant was for GN (between 1994 and 1999) and who on maintenance steroids In all 3 groups, the 4-year patient and graft survival rate, ARfree survival rate, serial annual serum creatinine level, and estimated GFR were similar. Although longer follow-up is needed, Ibrahim et al concluded that RDP conferred no increased short-term risks for recipients with GN. Likewise, Boardman et al noted no difference in outcome for recipients with FSGS who underwent RDP vs. chronic steroid therapy (73).

d. High immunologic risk - Khwaja et al. studied outcome for 78 recipients with a high immunologic risk recipients and on ATG, CSA-MMF, and RDP (74). The actuarial 3-year patient survival rate was 95\%; graft survival, 94\%. Mean serum creatinine level $( \pm \mathrm{SE})$ was $1.7 \pm 0.6$ at 6 months, $1.5 \pm 0.5$ at 12 months, and $1.6 \pm 0.7$ at 24 months. At the time of the report, only 2 had had biopsy-proven AR and 79\% remained prednisone-free. In a pilot study, Alloway et al. treated 10 high-risk recipients with dacluzimab induction, TAC-MMF, and RDP (75); because of high a AR rate (60\%), induction was switched to ATG, and AR rates subsequently were $27 \%$.

e. Kidney pancreas recipients- Canterovich et al. (ATG, CSA-MMF) and Kaufman et al. (ATG, TAC-MMF) have reported excellent graft survival rates and low AR rates in simultaneous kidney-pancreas (SKP) recipients on RDP (76,77). In a protocol using ATG and RDP, Gallon et al. showed no difference in outcome for SPK recipients on TAC-MMF vs. TAC-SRL (85). Kaufman et al. subsequently showed that Alemtuzumab and ATG were associated with equivalent results in their RDP protocol with TAC-SRL maintenance (86). Recently, Rajab et al. compared outcome for 97 pancreas recipients (80\% SPK; $20 \%$ pancreas after kidney transplants) (ATG, CSA-SRL, RDP) vs. historical controls (basiliximab, MMF, and maintenance prednisone (87): the patient and graft survival rates did not 
differ between the 2 groups; however, the RDP group had significantly lower AR rates $(\mathrm{p}<.01)$. In a recent randomized study Canterovich et al. compared RDP vs. prednisone withdrawal for SPK recipients on ATG, CSA-MMF (88). The 2 groups did not differ in 1-year patient and graft survival or AR rates; however, serum creatinine level was higher in the RDP group ( $\mathrm{p}=.02)$.

f. ABO-incompatible transplant recipients-we know of only 1 report of RDP in ABO-incompatible recipients. Kato et al. noted that the success of RDP (as defined by the need to reintroduce steroids) was significantly lower in ABO-incompatible (vs. ABO-compatible) recipients (51).

\section{Which immunosuppressive protocol for RDP?}

\section{a) induction}

Most RDP protocols have used induction therapy; when induction therapy was not used, the AR rates were high $(8,35,43)$. In a randomized controlled trial of CSA monotherapy vs. basiliximab-CSA, Parrot et al. found that significantly more basiliximab-CSA-treated recipients remained prednisone-free ( $\mathrm{p}=.046)(8)$. Vitco et al noted a high AR rate $(26.1 \%)$ in their basiliximab-TAC group (35). Boots et al. had a 29\% AR rate in their TAC-MMF group (43). No prospective randomized trials have compared induction agents in RDP protocols. Success has been reported with IL-2R antagonists,, Alemtuzumab,, and ATG.. However, in a randomized study of RDP vs. prednisone in which institutions could choose which antibody to use, Woodle et al. noted less AR with ATG (vs. IL-2R antagonists) (39).

\section{b) maintenance}

Kumar et al. randomized 150 nonsensitized patients treated with basiliximab and RDP to either TAC-MMF vs. TAC- SRL (82). The mean reported follow-up was $429 \pm 301$ days, with a minimum of 6 months. There was no significant difference between the groups in 2year patient and graft survival, or AR rates. Of note, however, surveillance biopsies performed during their study showed a lower incidence of subclinical AR (16\% vs. $27 \%)$ and chronic allograft nephropathy (10\% vs. $22 \%$ ) in the TAC-SRL group compared with the TAC-MMF group.

At our institution, Kandaswamy et al. randomized 450 first and second kidney recipients on 5 days of ATG and RDP to 1 of 3 groups: CSA-MMF; high-level TAC (8-12 ng/mL) and low-level SRL (3-7 ng/mL); low-level TAC (3-7 ng/mL) and high-level SRL (8-12 ng/mL) $(82,83)$. At 4 years posttransplant, patient, graft, death-censored graft, or AR-free graft survival rates, or in renal function did not differ significantly. But the 2 TAC-SRL groups had a higher rate of new onset diabetes.

Gallon et al. randomized recipients on IL-2R antibody and RDP posttransplant to TACMMF ( $n=45)$ vs. TAC-SRL ( $n=37)(84)$. At 3 years posttransplant, the graft survival was significantly better in the TAC-MMF group (1 graft lost) vs. TAC-SRL (6 grafts lost) $(\mathrm{p}=0.04)$. But the AR rate did not differ. Of note, $70 \%$ of the AR episodes occurred early ( $<30$ days posttransplant) in both groups, suggesting induction therapy using IL-2RA in RDP protocols may be ineffective. The slope of GFR decline per month was flatter in the TAC-MMF than in the TAC-SRL group. As described above, the same group compared outcome for SPK recipients treated with ATG, RDP, and either TAC-MMF vs. TAC-SRL (78). At 6 years, they found no difference between groups in patient or graft survival or in AR rates. Importantly, the slope of GFR decline per month at 5 years did not differ between the 2 groups.

Thus, studies to date suggest that excellent patient and graft survival rates and low AR rates can be obtained using a variety of maintenance drugs in RDP protocols. 


\section{AR after RDP: the question of long-term prednisone}

One clinically important question is whether long-term maintenance steroids should be introduced in recipients who have an AR episode after RDP. Humar et al. reported on 149 recipients who had $\geq 1$ AR episode while on our RDP protocol. AR episodes were treated with a steroid taper (with or without antibody) (85). Of the 149 recipients, 51 (34\%) switched to maintenance prednisone (5 mg/day) after treatment of their first AR episode; 98 (66\%) were returned to a steroid-free protocol. Return to maintenance prednisone was not randomized but was based, in part, on physician and patient choice. Patient characteristics for the two groups were similar. At a mean follow-up of 26 months, $32 \%$ of the recipients had a second AR episode: $29.4 \%$ of those on maintenance steroids vs. $33.7 \%$ of those remaining steroid free $(p=0.12)$. Graft survival was not significantly different between the 2 groups. Multivariate analysis of risk factors for a 2 nd episode suggested that whether or not steroids had been added to the maintenance protocol might have an impact $(\mathrm{RR}=2.1, \mathrm{p}$ $=0.07$ ). Of concern, in the subgroup supposedly most likely to not have a second AR episode - i.e., recipients with minimal to mild AR, the rate of second AR episodes significantly increased if the recipient had returned to steroid-free immunosuppression ( $\mathrm{p}=$. 02 ). Clearly, a randomized trial with longer follow-up and more recipients is necessary to definitively answer this question.

\section{Conclusions}

The potential benefit of eliminating steroid-related side effects for transplant recipients is obvious. Yet concerns remain that steroid-free maintenance immunosuppression protocols will have some long-term detrimental effects. It will be difficult to design studies to address such concerns. In the last decade, partly as a result of trials focused on late steroid withdrawal and RDP, recipients maintained on prednisone are taking far less prednisone than they would have been taking 10 years ago. The ideal study would be to compare RDP with a protocol involving rapid tapering to $5 \mathrm{mg} / \mathrm{day}$, and with a protocol involving rapid tapering plus late withdrawal. Currently, early transplant results are so good that the "n" required to power such a study would be enormous.

Clearly, some steroid-related side effects can occur early and with relatively low doses of steroids; the major benefit of a new randomized study would be to determine whether longterm low-dose prednisone has any salutary effects. And it might be important to do such a study with the "correct" antibody or maintenance therapy. The randomized study of Woodle et al. suggested - not statistically significant - that ATG was associated with fewer early AR episodes than an Il-2R receptor antagonist (39).

The popularity of RDP protocols nowadays has other implications. The long-term, low-dose study contemplated above would likely be difficult to do at institutions already using RDP, since their recipients would want to be on an RDP protocol (rather than on a study of RDP). In the United States, use of RDP protocols is growing (86). Yet steroid-free immunosuppression is not approved by the FDA. Therefore, any new drug trials must use prednisone in a comparison group. As a consequence, many recipients at institutions using RDP have no interest in participating that might randomize them to long-term prednisone.

An interesting question is why RDP is not, in most studies, associated with an increased AR rate, whereas steroid withdrawal at 3 months posttransplant - using the same maintenance immunosuppression - is. Part of the answer might be that cytokine receptor expression is increased by glucocorticoid-pretreated T cells (87); doing so, in turn, enhances IL-2stimulated T-cell proliferation. Clinically, proliferation may translate into enhanced T-cell activity when steroids are slowly withdrawn, as opposed to rapidly eliminated, and the result 
is a higher AR rate. Of interest, steroids decrease the bioavailability of MMF by increasing hepatic UDP-glucuronyl transferase activity. One study showed that, when steroids were tapered or withdrawn, the MMF AUC increased (88); thus there was more MMF exposure, possibly resulting in less AR. Another study showed that TAC exposure also increased after steroid withdrawal (89).

A final question is how to balance steroid-free and CNI-free approaches. Steroid-free immunosuppression has the obvious advantages of eliminating steroid-related side effects. But numerous studies have now demonstrated better long-term kidney allograft function when the use of CNIs is either minimized or eliminated. The ideal would be to develop protocols that are both steroid- and CNI-free; to date, however, such protocols have been associated with significant side effects. Hopefully, development of newer immunosuppressive drugs will permit long-term effective immunosuppression without side effects.

\section{Acknowledgments}

Supported by NIH grant \#: DK13083

I would like to thank Mary Knatterud for editorial assistance and Stephanie Daily and Barb Bailey for preparation of the manuscript.

\section{References}

1. Citterio F. Steroid side effects and their impact on transplantation outcome. Transplantation. 2001; 72:S75. [PubMed: 11833145]

2. Vanrenterghem YF, Claes K, Montagnino G, et al. Risk factors for cardiovascular events after successful renal transplantation. Transplantation. 2008 Jan 27.85:209. [PubMed: 18212625]

3. Veenstra DL, Best JH, Hornberger J, Sullivan SD, Hricik DE. Incidence and long-term cost of steroid-related side effects after renal transplantation. Am J Kidney Dis. 1999; 33(5):829-39. [PubMed: 10213637]

4. Schweizer RT, Rovelli M, Palmeri D, Vossler E, Hull D, Bartus S. Noncompliance in organ transplant recipients. Transplantation. 1990; 49:374. [PubMed: 2305467]

5. Nevins TE, Kruse L, Skeans MA, Thomas W. The natural history of azathioprine compliance after renal transplantation. Kidney Int. 2001; 60:1565. [PubMed: 11576374]

6. Prasad GV, Nash MM, McFarlane PA, Zaltzman JS. Renal transplant recipient attitudes toward steroid use and steroid withdrawal. Clin Transplant. 2003; 17:135. [PubMed: 12709080]

7. Montagnino G, Tarantino A, Maccario M, Elli A, Cesana B, Ponticelli C. Long-term results with cyclosporine monotherapy in renal transplant patients: a multivariate analysis of risk factors. Am $\mathrm{J}$ Kidney Dis. 2000; 35:1135. [PubMed: 10845828]

8. Parrott NR, Hammad AQ, Watson CJE, Lodge JPA, Andrews CD. Multicenter, randomized study of the effectiveness of basiliximab in avoiding addition of steroids to cyclosporine A monotherapy in renal transplant recipients. Transplantation. 2005; 79:344. [PubMed: 15699766]

9. Hricik DE, O’Toole MA, Schulak JA, Herson J. Steroid-free immunosuppression in cyclosporinetreated renal transplant recipients: a meta-analysis. J Am Soc Nephrol. 1993; 4 :1300. [PubMed: 8130356]

10. Kasiske BL, Chakkera HA, Louis TA, Ma JZ. A meta-analysis of immunosuppression withdrawal trials in renal transplantation. Am Soc Nephrol. 2000; 11:1910.

11. Sinclair NR. Low-dose steroid therapy in cyclosporine-treated renal transplant recipients with wellfunctioning grafts. The Canadian Multicentre Transplant Study Group. CMAJ. 1992; 147:645. [PubMed: 1521210]

12. Almond PS, Matas A, Gillingham K, et al. Risk factors for chronic rejection in renal allograft recipients. Transplantation. 1993; 55:752. [PubMed: 8475548] 
13. Ahsan N, Hricik D, Matas A, et al. Prednisone withdrawal in kidney transplant recipients on cyclosporine and mycophenolate mofetil -- a prospective randomized study. Steroid Withdrawal Study Group. Transplantation. 1999; 68:1865. [PubMed: 10628766]

14. Vanrenterghem Y, Lebranchu Y, Hene R, Oppenheimer F, Ekberg H. Double-blind comparison of two corticosteroid regimens plus mycophenolate mofetil and cyclosporine for prevention of acute renal allograft rejection. Transplantation. 2000; 70:1352. [PubMed: 11087152]

15. Smak Gregoor PJH, De Sévaux RGL, Ligtenberg G, et al. Withdrawal of cyclosporine or prednisone six months after kidney transplantation in patients on triple drug therapy: a randomized, prospective, multicenter study. J Am Soc Nephrol. 2002; 13:1365. [PubMed: 11961025]

16. Boletis JN, Konstadinidou I, Chelioti H, et al. Successful withdrawal of steroid after renal transplantation. Transplant Proc. 2001; 33:1231. [PubMed: 11267272]

17. Squifflet JP, Vanrenterghem, van Hooff JP, Salmela K, Rogitti P. the European Tacrolimus/MMF Transplantation Study Group. Safe withdrawal of corticosteroids or mycophenolate mofetil: results of a large, prospective, multicenter, randomized study. Transplant Proc. 2002; 34:1584. [PubMed: 12176495]

18. Solá E, Alférez MJ, Cabello M, et al. Low-dose and rapid steroid withdrawal in renal transplant patients treated with tacrolimus and mycophenolate mofetil. Transplant Proc. 2002; 34:1689. [PubMed: 12176537]

19. Pascual J, Quereda C, Zamora J, Hernández D. Spanish Group for Evidence-Based Medicine in Renal Transplantation. Steroid withdrawal in renal transplant patients on triple therapy with a calcineurin inhibitor and mycophenolate mofetil: a meta-analysis of randomized, controlled trials. Transplantation. 2004; 78:1548. [PubMed: 15599321]

20. Pascual J, Quereda C, Zamora J, Hernández D. Spanish Group for Evidence-Based Medicine in Renal Transplantation. Updated metaanalysis of steroid withdrawal in renal transplant patients on calcineurin inhibitor and mycophenolate mofetil. Transplant Proc. 2005; 37:3746. [PubMed: 16386525]

21. Pascual J, van Hooff JP, Salmela K, Lang P, Rigotti P, Budde K. on behalf of all participating investigators. Three-year observational follow-up of a multicenter, randomized trial on tacrolimusbased therapy with withdrawal of steroids or mycophenolate mofetil after renal transplant. Transplantation. 2006; 82:55. [PubMed: 16861942]

22. Opelz G, Döhler B, Laux G. for the Collaborative Transplant Study. Long-term prospective study of steroid withdrawal in kidney and heart transplant recipients. Am J Transplant. 2005; 5:720. [PubMed: 15760395]

23. Pelletier RP, Akin B, Ferguson RM. Prospective, randomized trial of steroid withdrawal in kidney recipients treated with mycophenolate mofetil and cyclosporine. Clin Transplant. 2006; 20:10. [PubMed: 16556147]

24. Wlodarczyk Z, Walaszewski J, Perner F, et al. Steroid withdrawal at 3 months after kidney transplantation: a comparison of two tacrolimus-based regimens. Transplant Int. 2005; 18:157.

25. Buchler M, Caillard S, Barbier S, et al. Sirolimus versus cyclosporine in kidney recipients receiving thymoglobulin, mycophenolate mofetil and a 6-month course of steroids. Am J Transplant. 2007; 7:2522. [PubMed: 17868057]

26. Mahalati K, Kahan BD. A pilot study of steroid withdrawal from kidney transplant recipients on sirolimus-cyclosporine a combination therapy. Transplant Proc. 2001; 33:3232. [PubMed: 11750385]

27. Citterio F, Sparacino V, Altieri P, et al. Addition of sirolimus to cyclosporine in long-term kidney transplant recipients to withdraw steroid. Transplant Proc. 2005; 37:827. [PubMed: 15848545]

28. Hricik DE, Knauss TC, Bodziak KA, et al. Withdrawal of steroid therapy in AA kidney transplant recipients receiving sirolimus and tacrolimus. Transplantation. 2003; 76:938. [PubMed: 14508357]

29. Hricik DE, Augustine JJ, Knauss TC, et al. Long-term graft outcomes after steroid withdrawal in AA kidney transplant recipients receiving sirolimus and tacrolimus. Transplantation. 2007; 83:277. [PubMed: 17297401] 
30. Birkeland SA. Steroid-free immunosuppression after kidney transplantation with antithymocyte globulin induction and cyclosporine and mycophenolate mofetil maintenance therapy. Transplantation. 2002; 73:1527. [PubMed: 12023637]

31. Birkeland SA. Steroid-free immunosuppression in renal transplantation: a long-term follow-up of 100 consecutive patients. Transplantation. 2001; 71:1089. [PubMed: 11374407]

32. Vincenti F, Monaco A, Grinyo J, Kinkhabwala M, Roza A. Multicenter randomized prospective trial of steroid withdrawal in renal transplant recipients receiving basiliximab, cyclosporine microemulsion and mycophenolate mofetil. Am J Transplant. 2003; 3:306. [PubMed: 12614286]

33. ter Meulen CG, van Riemsdijk I, Hené RJ, et al. Steroid-withdrawal at 3 days after renal transplantation with anti-IL-2 receptor a therapy: a prospective, randomized, multicenter study. Am J Transplant. 2004; 4:803. [PubMed: 15084178]

34. Rostaing L, Cantarovich D, Mourad G, et al. Corticosteroid-free immunosuppression with tacrolimus, mycophenolate mofetil, and daclizumab induction in renal transplantation. Transplantation. 2005; 79:807. [PubMed: 15818323]

35. Vitko S, Klinger M, Salmela K. Two corticosteroid-free regimens - tacrolimus monotherapy after basiliximab administration and tacrolimus/mycophenolate mofetil - in comparison with a standard triple regimen in renal transplantation: results of the Atlas Study. Transplantation. 2005; 80:1734. [PubMed: 16378069]

36. Woodle ES. for the TRIMS Study Group. A randomized, prospective, multicenter comparative study evaluating a Thymoglobulin-based early corticosteroid cessation regimen in renal transplantation (TRIMS). World Transplant Congress. 2006:294.

37. Vincenti F, Schena FP, Paraskevas S, Hauser IA, Walker RG, Grinyo J. FREEDOM Study Group. A randomized, multicenter study of steroid avoidance, early steroid withdrawal or standard steroid therapy in kidney transplant recipients. Am J Transplant. 2008; 8:307. [PubMed: 18211506]

38. Laftavi MR, Stephan R, Stefanick B, et al. Randomized prospective trial of early steroid withdrawal compared with low-dose steroids in renal transplant recipients using serial protocol biopsies to assess efficacy and safety. Surgery. 2005; 137:364. [PubMed: 15746793]

39. Woodle ES. Astellas Steroid Withdrawal Study Group. A randomized double blind, placebocontrolled trial of early corticosteroid cessation versus chronic corticosteroids: three year results. Am J Transplant. 2006; 6:S177.

40. Montagnino G, Sandrini S, Iorio B, et al. A randomized exploratory trial of steroid avoidance in renal transplant patients treated with everolimus and low-dose cyclosporine. Nephrol Dial Transplant. 2008; 23:707. [PubMed: 17890244]

41. Kumar MS, Heifets M, Moritz MJ, Saeed MI, Khan SM, Fyfe B, Sustento-Riodeca N, Daniel JN, Kumar A. Safety and efficacy of steroid withdrawal two days after kidney transplantation: analysis of results at three years. Transplantation. 2006; 81:832. [PubMed: 16570004]

42. Kumar MSA, Heifets M, Fyfe B, et al. Comparison of steroid avoidance in tacrolimus/ Mycophenolate mofetil and tacrolimus/sirolimus combination in kidney transplantation monitored by surveillance biopsy. Transplantation. 2005; 80:807. [PubMed: 16210969]

43. Boots JM, Christiaans MHL, Van Duijnhoven EM, van Suylen RJ, van Hooff JP. Early steroid withdrawal in renal transplantation with tacrolimus dual therapy: a pilot study. Transplantation. 2002; 74:1703. [PubMed: 12499885]

44. Cole E, Landsberg D, David R, et al. A pilot study of steroid-free immunosuppression in the prevention of acute rejection in renal allograft recipients. Transplantation. 2001; 72:845. [PubMed: 11571448]

45. Rajab A, Pelletier RP, Henry ML, et al. Excellent clinical outcomes in primary kidney transplant recipients treated with steroid-free maintenance immunosuppression. Clin Transplant. 2006; 20:537. [PubMed: 16968478]

46. Woodle ES, Vincenti F, Lorber MI, et al. A multicenter pilot study of early (4-day) steroid cessation in renal transplant recipients under simulect, tacrolimus and sirolimus. Am J Transplant. 2005; 5:157. [PubMed: 15636625]

47. Kato Y, Tojimbara T, Iwadoh K, et al. Early steroid withdrawal protocol with basiliximab, cyclosporine and mycophenolate mofetil in renal-transplant recipients. Int Immunopharmacol. 2006; 6:1984. [PubMed: 17161352] 
48. Jaber JJ, Feustel PJ, Elbahloul O, et al. Early steroid withdrawal therapy in renal transplant recipients: a steroid-free sirolimus and CellCept-based calcineurin inhibitor-minimization protocol. Clin Transplant. 2007; 21:101. [PubMed: 17302598]

49. Matas AJ, Ramcharan T, Paraskevas S, et al. Rapid discontinuation of steroids in living donor kidney transplantation: a pilot study. Am J Transplant. 2001; 1:278-83. [PubMed: 12102262]

50. Humar A, Kandaswamy R, Payne W, et al. 6 Years of prednisone-free maintenance immunosuppression. Am J Transplant. 2006; 6:S482.

51. Matas AJ, Kandaswamy R, Humar A, et al. Long-term immunosuppression, without maintenance prednisone, after kidney transplantation. Ann Surg. 2004; 240:510. [PubMed: 15319722]

52. Gallon LG, Winoto J, Leventhal JR, et al. Effect of prednisone versus no prednisone as part of maintenance immunosuppression on long-term renal transplant function. Clin J Am Soc Nephrol. 2006; 1:1029. [PubMed: 17699323]

53. van den Ham EC, Kooman JP, Christiaans MH, Nieman FH, van Hooff JP. Weight changes after renal transplantation: a comparison between patients on 5-mg maintenance steroid therapy and those on steroid-free immunosuppressive therapy. Transplant Int. 2003; $16: 300$.

54. Mataunami C, Hilton AF, Dyer JA, Rumbach OW, Hardie IR. Ocular complications in renal transplant patients. Australian and New Zealand J of Ophthalmology. 1994; 22:53.

55. Julian BA, Laskow DA, Dubovsky J, Dubovsky EV, Curtis J, Quarles LD. Rapid loss of vertebral mineral density after renal transplantation. N Engl J Med. 1991; 325:544. [PubMed: 1857390]

56. Wolpaw T, Deal CL, Fleming-Brooks S, Bartucci MR, Schulak JA, Hricik DE. Factors influencing vertebral bone density after renal transplantation. Transplantation. 1994; 58:1186. [PubMed: 7992360]

57. Lo Cascio V, Bonucci E, Imbimbo B, Ballanti P, Tartarotti D, Galvanini G, et al. Bone loss after glucocorticoid therapy. Calcif Tissue Int. 1984; 36:435. [PubMed: 6435841]

58. Laan RF, van Riel PL, van de Putte LB, van Erning LJ, van't Hof MA, Lemmens JA. Low-dose prednisone induces rapid reversible axial bone loss in patients with rheumatoid arthritis. A randomized, controlled study. Ann Intern Med. 1993; 119:963-8. [PubMed: 8105737]

59. van Staa TP, Leufkens HG, Abenhaim L, Zhang B, Cooper C. Oral corticosteroids and fracture risk: relationship to daily and cumulative doses. Rheumatology (Oxford). 2000; 39:1383. [PubMed: 11136882]

60. Gluck OS, Murphy WA, Hahn TJ, Hahn B. Bone loss in adults receiving alternate day glucocorticoid therapy. A comparison with daily therapy. Arthritis Rheum. 1981; 24:892-8. [PubMed: 7259801]

61. Ruegsegger P, Medici TC, Anliker M. Corticosteroid-induced bone loss. A longitudinal study of alternate day therapy in patients with bronchial asthma using quantitative computed tomography. Eur J Clin Pharmacol. 1983; 25(5):615-20. [PubMed: 6662161]

62. Birkeland SA, Larsen KE, Rohr N. Pediatric renal transplantation without steroids. Pediatr Nephrol. 1998; 12:87. [PubMed: 9543361]

63. Sarwal MM, Yorgin PD, Alexander S, et al. Promising early outcomes with a novel, complete steroid avoidance immunosuppression protocol in pediatric renal transplantation. Transplantation. 2001; 72:13. [PubMed: 11468528]

64. Sarwal MM, Vidhun JR, Alexander SR, et al. Continued superior outcomes with modification and lengthened follow-up of a steroid-avoidance pilot with extended daclizumab induction in pediatric renal transplantation. Transplantation. 2003; 76:1331. [PubMed: 14627912]

65. Oberholzer J, John E, Lumpaopong A, et al. Early discontinuation of steroids is safe and effective in pediatric kidney transplant recipients. Pediatr Transplantation. 2005; 9:456.

66. Chavers BM, Chang C, Gillingham KJ, Matas AJ. Successful pediatric kidney transplantation using a novel protocol of rapid discontinuation of prednisone. J Am Soc Nephrol (Abstracts Issue). 2006; 17:672A.

67. Haririan A, Sillix DH, Morawski K, et al. Short-term experience with early steroid withdrawal in African-American renal transplant recipients. Am J Transplant. 2006; 6 :2396. [PubMed: 16869806] 
68. Boardman RE, Alloway RR, Alexander JW, et al. African-American renal transplant recipients benefit from early corticosteroid withdrawal under modern immunosuppression. Am J Transplant. 2005; 5:356. [PubMed: 15643996]

69. Anil Kumar MS, Moritz MJ, Saaed MI, et al. Avoidance of chronic steroid therapy in AA kidney transplant recipients monitored by surveillance biopsy: 1-year results. Am J Transplant. 2005; 5:1976. [PubMed: 15996248]

70. Zeng X, El-Amm JM, Doshi MD, et al. Intermediate-term outcomes with early steroid withdrawal in African-American renal transplant recipients undergoing surveillance biopsy. Surgery. 2007; 142:538. [PubMed: 17950346]

71. Anil Kumar MS, Khan S, Ranganna K, Malat G, Sustento-Reodica N, Meyers WC. Long-term outcome of early steroid withdrawal after kidney transplantation in African American recipients monitored by surveillance biopsy. Am J Transplant. 2008; 8:574. [PubMed: 18294153]

72. Ibrahim H, Rogers T, Casingal V, et al. Graft loss from recurrent glomerulonephritis is not increased with a rapid steroid discontinuation protocol. Transplantation. 2006; 81:214. [PubMed: 16436965]

73. Boardman R, Trofe J, Alloway R, et al. Early steroid withdrawal does not increase risk for recurrent focal segmental glomerulosclerosis. Transplant Proc. 2006; 37:817. [PubMed: 15848542]

74. Kwajha K, Asolati M, Harmon JV, et al. Rapid discontinuation of prednisone in higher-risk kidney transplant recipients. Transplantation. 2004; 78:1397. [PubMed: 15548981]

75. Alloway RR, Hanaway MJ, Trofe J, et al. A prospective, pilot study of early corticosteroid cessation in high-immunologic-risk patients: the Cincinnati experience. Transplant Proc. 2005; 37:802. [PubMed: 15848537]

76. Cantarovich D, Giral-Classe M, Hourmant M, et al. Low incidence of kidney rejection after simultaneous kidney-pancreas transplantation after antithymocyte globulin induction and in the absence of corticosteroids: results of a prospective pilot study in 28 consecutive cases. Transplantation. 2000; 69:1505. [PubMed: 10798780]

77. Kaufman DB, Leventhal JR, Koffron AJ, et al. A prospective study of rapid corticosteroid elimination in simultaneous pancreas-kidney transplantation: comparison of two maintenance immunosuppression protocols: tacrolimus/mycophenolate mofetil versus tacrolimus/sirolimus. Transplantation. 2002; 73:169. [PubMed: 11821726]

78. Gallon LG, Winoto J, Chhabra D, et al. Long-term renal transplant function in recipient of simultaneous kidney and pancreas transplant maintained with two prednisone-free maintenance immunosuppressive combinations: tacrolimus/mycophenolate mofetil versus tacrolimus/sirolimus. Transplantation. 2007; 83:1324. [PubMed: 17519781]

79. Kaufman DB, Leventhal JR, Gallon LG, Parker MA. Alemtuzumab induction and prednisone-free maintenance immunotherapy in simultaneous pancreas-kidney transplantation comparison with rabbit antithymocyte globulin induction-long-term results. Am J Transplant. 2006; 6:331. [PubMed: 16426317]

80. Rajab A, Pelletier RP, Ferguson RM, Elkhammas EA, Bumgardner GL, Henry ML. Steroid-free maintenance immunosuppression with Rapamune and low-dose neoral in pancreas transplant recipients. Transplantation. 2007; 84:1131. [PubMed: 17998868]

81. Cantarovitch D, Karam G, Hourmant M, et al. Steroid avoidance versus steroid withdrawal after simultaneous pancreas-kidney transplantation. Am J Transplant. 2005; 5:1332. [PubMed: 15888038]

82. Anil Kumar MS, Heifets M, Fyfe B, et al. Comparison of steroid avoidance in tacrolimus/ mycophenolate mofetil and tacrolimus/sirolimus combination in kidney transplantation monitored by surveillance biopsy. Transplantation. 2005; 80:807. [PubMed: 16210969]

83. Kandaswamy R, Melancon JK, Dunn T, et al. A prospective randomized trial of steroid-free maintenance regimens in kidney transplant recipients - an interim analysis. Am J Transplant. 2005; 5:1529. [PubMed: 15888064]

84. Gallon L, Perico N, Dimitrov BD, et al. Long-term renal allograft function on a tacrolimus-based, pred-free maintenance immunosuppression comparing sirolimus versus MMF. Am J Transplant. 2006; 6:1617. [PubMed: 16827862] 
85. Humar A, Gillingham K, Kandaswamy R, Payne W, Matas A. Steroid avoidance regimens: a comparison of outcomes with maintenance steroids versus continued steroid avoidance in recipients having an acute rejection episode. Am J Transplant. 2007; 7:1948. [PubMed: 17617858]

86. USRDS 2007 Annual Data Report: Atlas of chronic kidney disease \& end-stage renal disease in the United States Transplantation. Am J Kid Dis. 2008; 51:S155-173.

87. Almawi WY, Melemedjian OK, Rieder MJ. An alternate mechanism of glucocorticoid antiproliferative effect: promotion of a Th2 cytokine-secreting profile. Clin Transplant. 1999; 13 :365. [PubMed: 10515216]

88. Cattaneo D, Perico N, Gaspari F, Gotti E, Remuzzi G. Glucocorticoids interfere with mycophenolate mofetil bioavailability in kidney transplantation. Kidney Int. 2002; 62:1060. [PubMed: 12164891]

89. van Duijnhoven EM, Boots JM, Christiaans MH, Stolk LM, Undre NA, van Hooff JP. Increase in tacrolimus trough levels after steroid withdrawal. Transpl Int. 2003; 16:721. [PubMed: 12827231] 


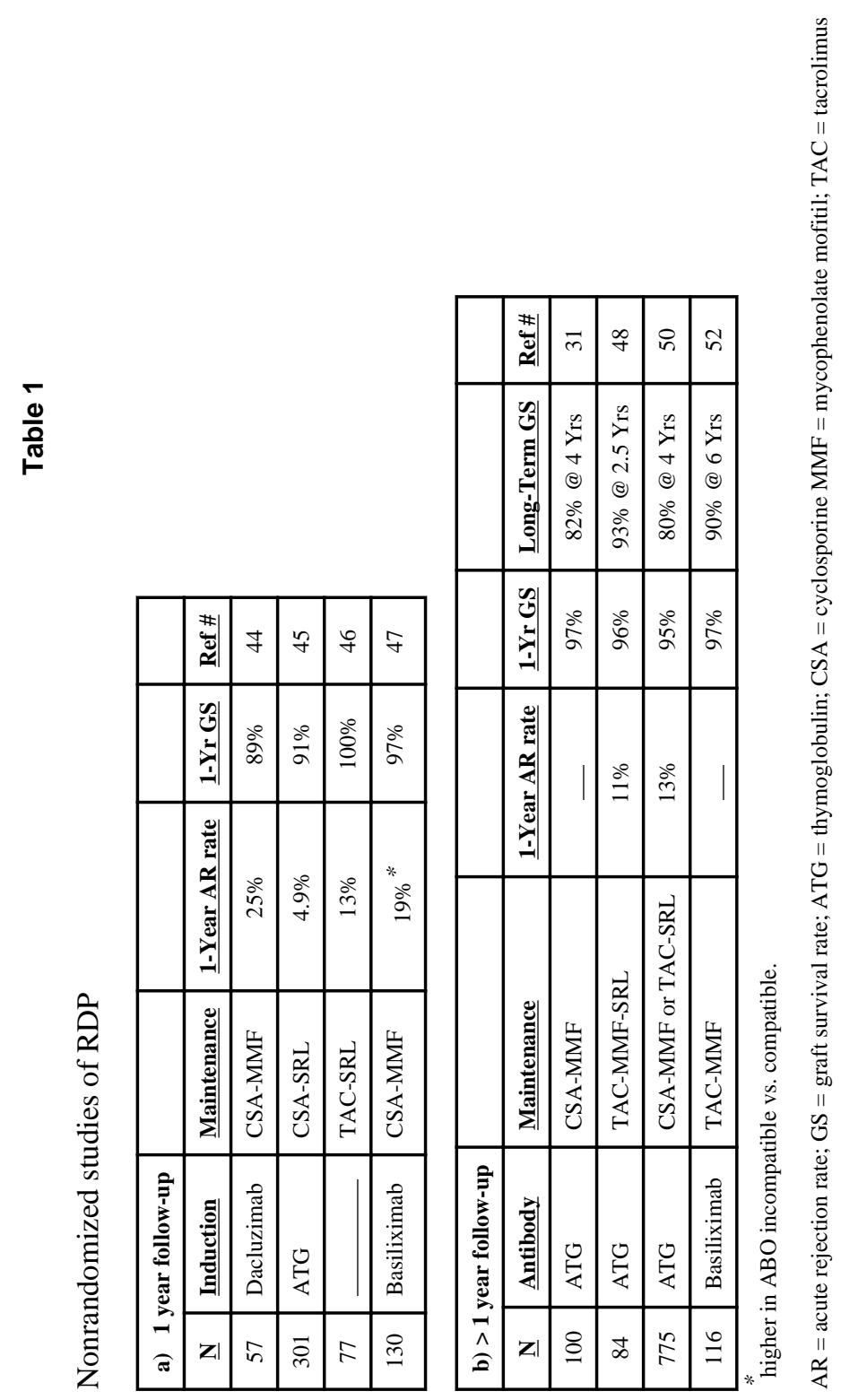

Transpl Int. Author manuscript; available in PMC 2013 January 24. 


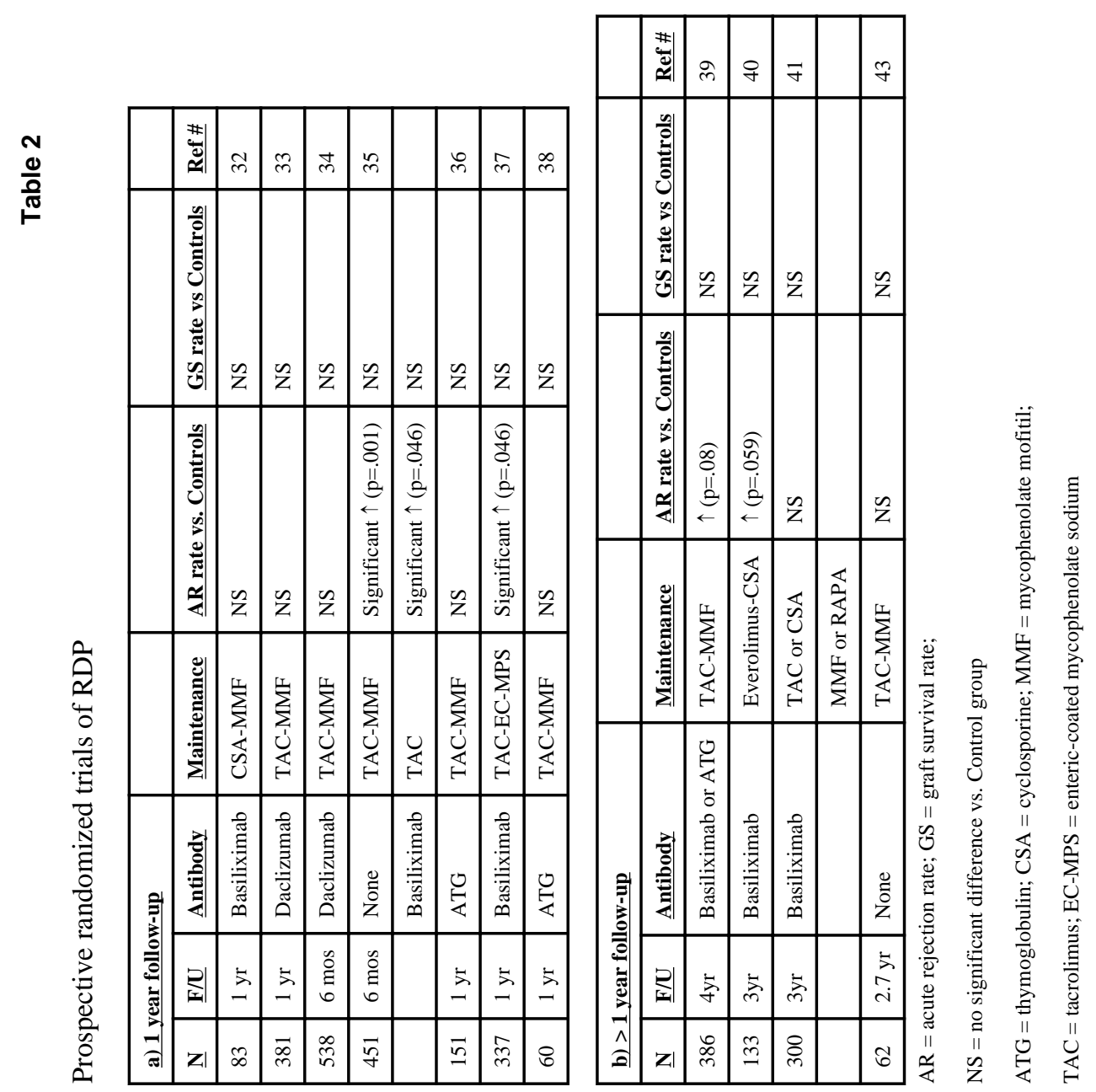

